\title{
Massive cutaneous complications due to snake bite: A rare case and literature review
}

\author{
Mahdieh Sadeghi ${ }^{1}$, Maryam Barazandeh $^{1}$, Zakaria zakariaei ${ }^{1}$, Lotfollah Davoodi ${ }^{1}$, Rabeeh \\ Tabaripour $^{1}$, Mahdi Fakhar ${ }^{1}$, and Ashkan Zakariaei ${ }^{1}$ \\ ${ }^{1}$ Mazandaran University of Medical Sciences
}

January 28, 2021

\begin{abstract}
Snake bite is a common and very important issue threatening health worldwide. Patients who develop hemorrhagic or nonhemorrhagic blisters following snakebite, in addition to anti-venom, need broad-spectrum antibiotics and sometimes emergency surgical procedures and hospitalization to prevent serious complications such as necrotizing fasciitis, compartment syndrome.
\end{abstract}

\section{Introduction:}

Snake bite is a common and very important issue threatening health in different parts of the world, including Iran [1]. The clinical manifestations of snake bites are varying and depend on many factors, including the species of snake, the amount and strength of the venom injected, the location of the bite, and the patient's own factors, such as age, the underlying disease [2].

Wound infections following snake bites such as cellulitis and necrotizing fasciitis, although not common, [3-5] are seen in the bites of snakes and if untreated can cause serious complications such as necrotizing fasciitis, extensive local tissue damage and progressive sepsis that can be associated with acute renal failure (ARF), thrombocytopenia, and coagulopathy; [2] although standard anti-venom treatment can reduce the toxic hemorrhagic or neurotoxic effects of venom, [6-8] the progression of compartment syndrome and bacterial tissue infection cannot be prevented by anti-venom treatment $[9,10]$ and requires emergency surgical procedures such as fasciotomy or amputation and broad-spectrum antibiotics and hospitalization in the intensive care unit $[3,7,11,12]$.

On the other hand, in some cases of snake bites, the patient does not notice the bite and after a while suffers from local symptoms and sometimes systemic complications that if not diagnosed and treated in time can lead to massive complications and injuries that will complicate the treatment. Thus, we describe a rare case of wound infection with hemorrhagic and non-hemorrhagic blisters following snakebite.

\section{Case presentation}

This study was conducted according to the declaration of Helsinki principles. Also, guidelines and methodology have been followed in this study. A 65-year-old man referred to the Cardiovascular Center following chest pain with dissemination to the left upper extremity, cold sweats, chills, his had nausea and vomiting, and edema in the left upper extremity. At the time of entering the Cardiovascular Center, the patient had no fever and blood pressure was $113 / 68 \mathrm{mmHg}$, pulse rate was 76 beat $/ \mathrm{min}$ and respiratory rate was 20 breaths/min. In examination, the ECG had a normal sinus rhythm and showed no changes indicative myocardial infarction. Blood samples were also taken to test for cardiac enzymes, Troponin $<0.2$ and BUN $=46 \mathrm{mg} / \mathrm{dL}$ and Creatinine $=2.5 \mathrm{mg} / \mathrm{dL}$. After 6 hours, the swelling of the left upper limb progressed and multiple blisters appeared, after which the patient was referred to an infectious center in the northern 
Iran for examination for infection, necrotizing fasciitis and compartment syndrome. After admission to the infectious center, the patient had a fever $(\mathrm{T}=38.5)$ and tachycardia $(\mathrm{PR}=108 / \mathrm{min})$ with decreased level of consciousness. In the emergency room of the infectious center, first the patient's airway checked out and to monitor his vital signs, cardiac monitoring and pulse oximetry along with oxygen therapy with $\mathrm{O}_{2}$ mask were performed. Blood pressure and respiratory rate were within normal limits, and examination of the lungs, abdomen, and central nervous system showed no abnormal findings. On examination of the left upper limb; there was erythema, warmness, stiffness, non-pitting edema, and limitation of active movement. Also, vesiculobullous lesions were seen on the dorsal surface of the hand to the wrist and both flexor and extensor surfaces of the forearm and arm up to the proximal arm and left axillary area. (figure 1-A, B)

The location and size of the axillary lymph nodes were normal and the left radial pulse was weakly palpable. Also Swelling, redness, and localized infection were seen in the distal third finger of the left hand, and according to the patient's history of burning sensation in the same finger after lifting a heavy object at home 3 days ago and also the endemicity of his place of habitation raised the probability of a venomous snake bite, and for this reason the patient was transferred to the poisoning ward.

The patient had no history of smoking or drug usage, his had a history of dyslipidemia, hypertension, diabetes mellitus and ischemic heart disease, which had been controlled with appropriate medications and was asymptomatic in routine life.

He also had no history of asthma, allergies, rhinitis, dermatitis or eczema and did not have a history of similar bites in the past.

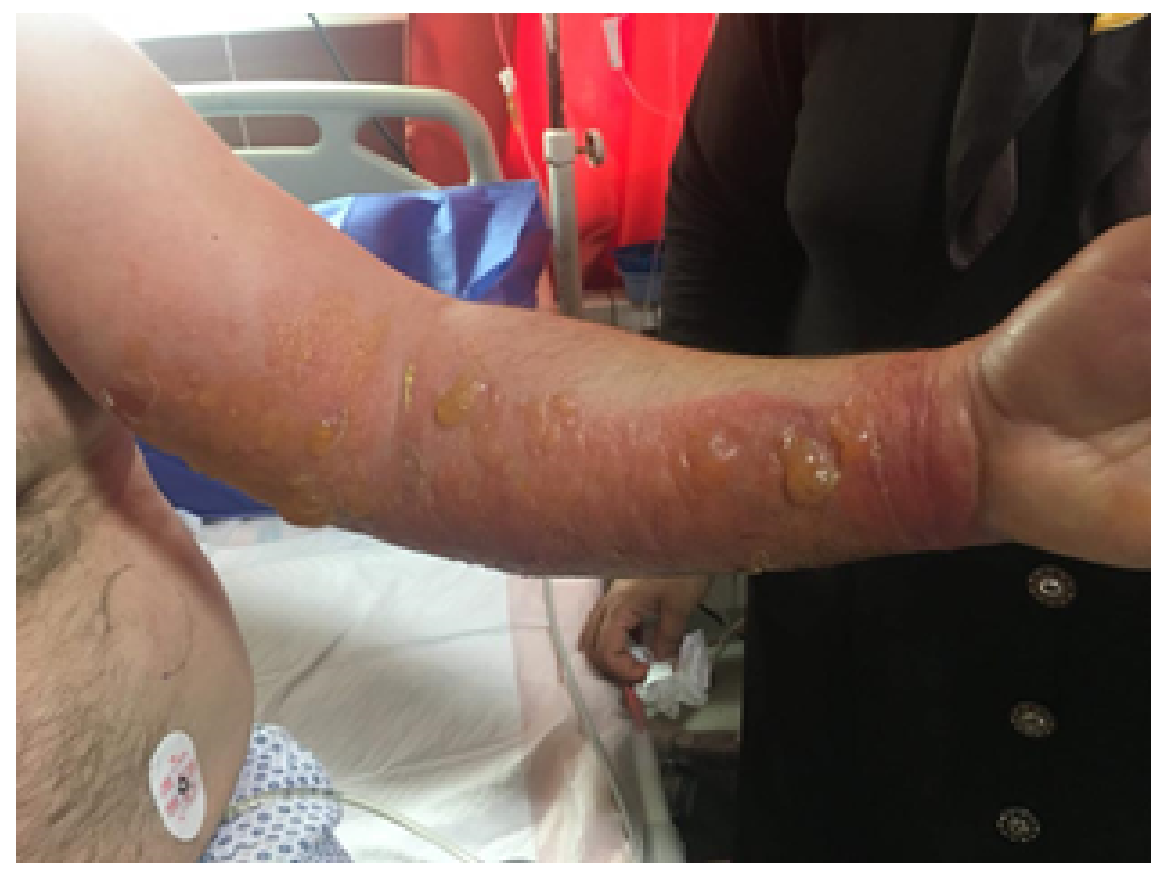




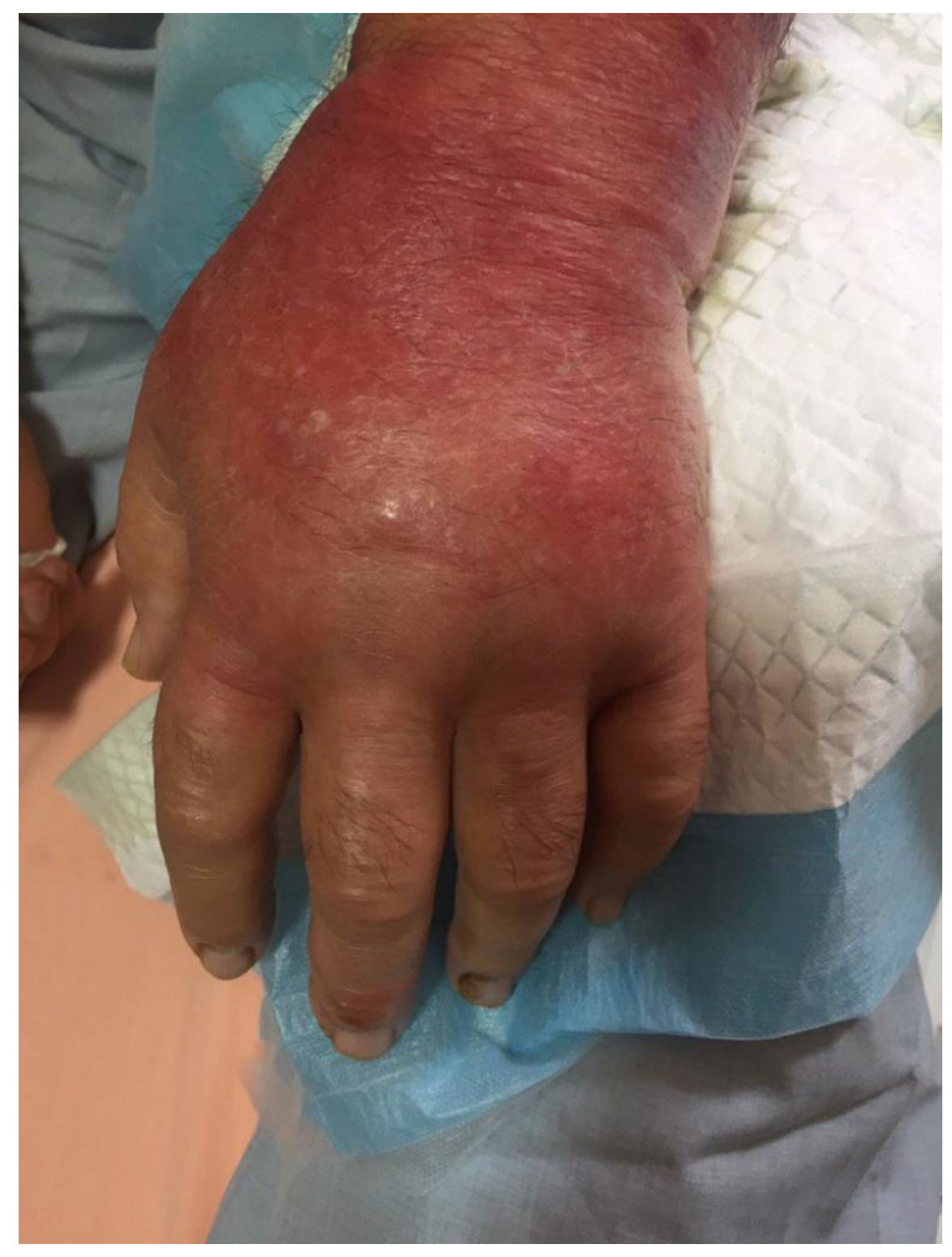




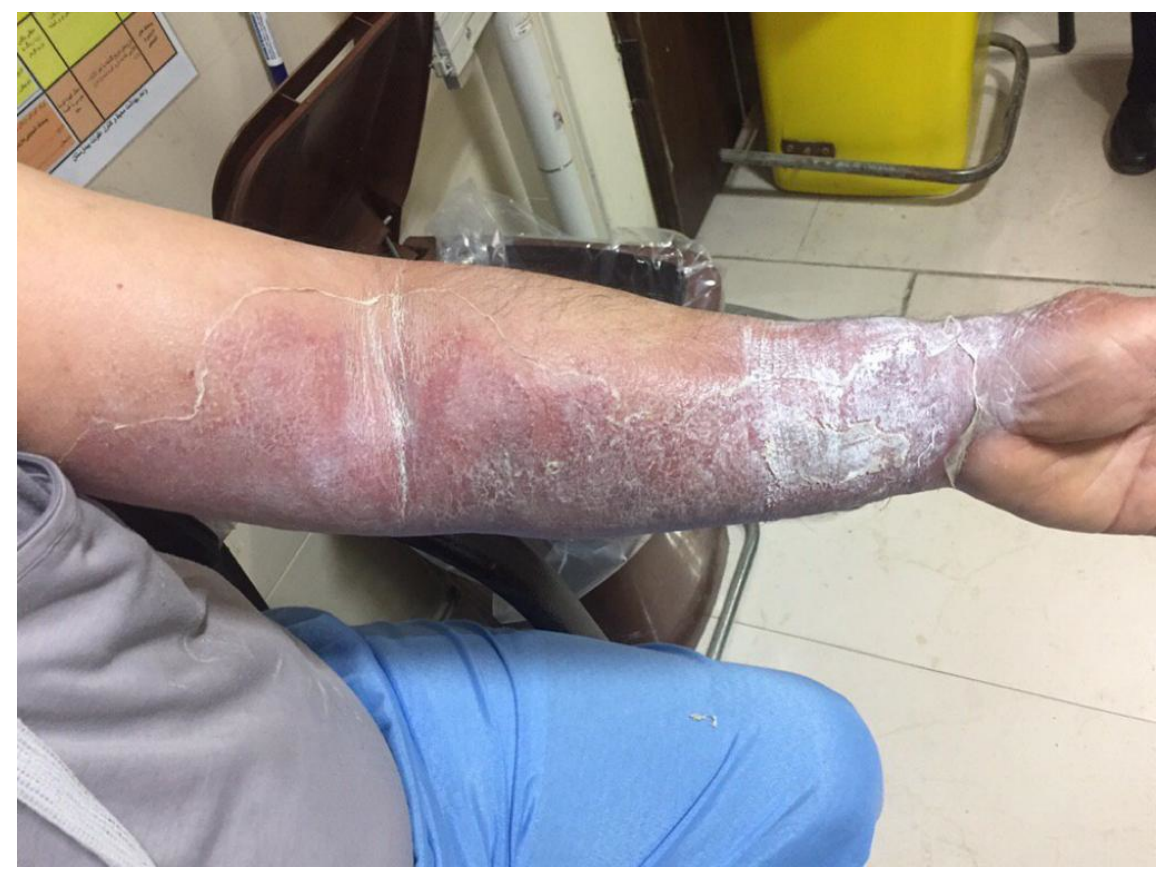




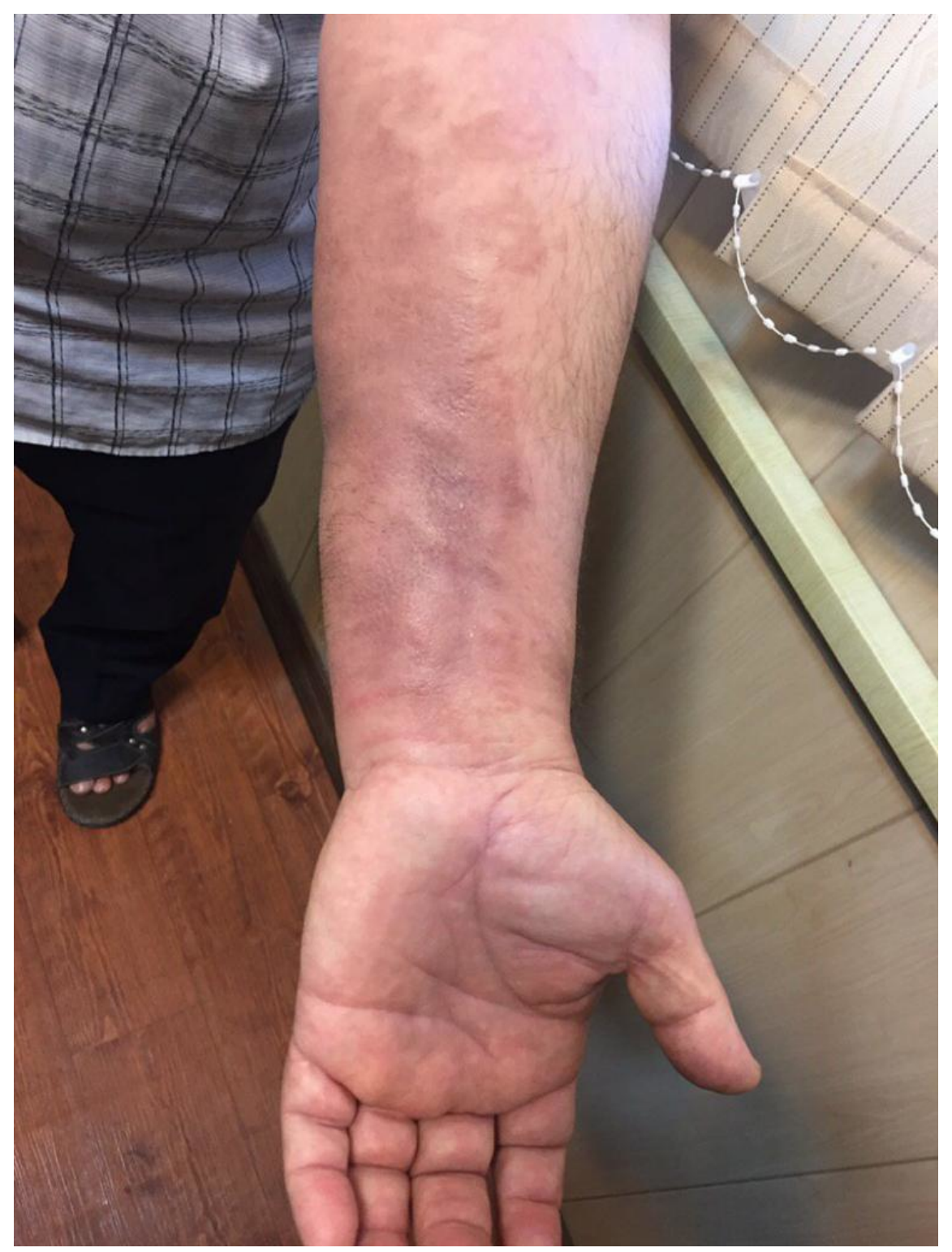

Figure 1. Vesiculobullous lesions before treatment (A and B) and after treatment (C and D)

In the primary tests performed; BUN and Creatinine increased compared to the measured values in the Heart Center, there was also a decrease in platelet count and a prolongation of PT and PTT, but the patient had no evidence of hemorrhage and coagulopathy, and liver enzymes were within the normal range (table 1); also, color doppler ultrasound of the arteries and veins of the left upper extremity did not provide evidence of thrombosis. The ultrasound also showed multiple fluid-containing tracts, and according to surgical and orthopedic consultation, there was no evidence of necrotizing fasciitis or compartment syndrome.

Table 1. Results of initial tests and comparison with measured values in the heart center

\begin{tabular}{llll}
\hline Parameter & Normal range & Blood sample in Heart center & Initial blood sample in Emergency room \\
\hline BUN & $13-43 \mathrm{mg} / \mathrm{dL}$ & 46 & 172 \\
Creatinine & $0.6-1.2 \mathrm{mg} / \mathrm{dL}$ & 2.5 & 3.3
\end{tabular}




\begin{tabular}{llll}
\hline Parameter & Normal range & Blood sample in Heart center & Initial blood sample in Emergency room \\
\hline PT & Control: 12 & 20 & 22.1 \\
PTT & & 32 & 54 \\
INR & & 1.7 & 2.7 \\
Platelet & $150000-450000 / \mu \mathrm{L}$ & $\mathrm{N} / \mathrm{A}$ & $104 \times 10^{3}$ \\
\hline
\end{tabular}

Abbreviation: $\mathrm{N} / \mathrm{A}=$ not available

On the first day of hospitalization, with the possibility of viral infection, was prescribed Acyclovir tablet $400 \mathrm{mg}$ Tds, and for bacterial infection, Ceftriaxone vial $1 \mathrm{gr} \mathrm{Bd}$, Teicoplanin (Targocid) $400 \mathrm{mg} \mathrm{Bd}$ and Meropenem 1 gr Bd intravenous infusion and as well as with probability of snakebite, 5 vials of antivenom via intravenous infusion, then 6 hours after the first injection, received 5 vials of antivenom and also 2 vials of antivenom every 6 hours in three times as a maintenance dose along with the medications he was already taking. The left upper limb was elevated and a hot water bag was used to reduce stiffness and edema in blister-free areas.

As for the increase in BUN and creatinine in the patient's blood sample, nephrology consultation was requested. Also following receiving antivenom, decrease in BUN and Creatinine levels and normalization of the level of consciousness, the diagnosis of acute interstitial nephritis (AIN) following snake bite was proposed.

On the second day, the Teicoplanin (Targocid) antibiotic was discontinued and Ciprofloxacin $200 \mathrm{mg}$ IV infusion was replaced. The wound was drained and then Eude Alibore solution, Silver Sulfadiazine, and Zinc Oxide ointments were also used for topical treatment of blisters.

The patient was monitored in the poisoning ward for 3 weeks, after which the fever, hematoma, and skin lesions resolved; the radial pulse is fully and symmetrical in the involved limb. Also, serum levels of BUN, Creatinine, PT, PTT, and INR returned to normal range and the patient was discharged from the hospital by good general condition (figure 1-C, D). The patient gave verbally informed consent in this regard.

\section{Discussion}

Snake venom contains of various toxins that acting systemically and locally, including, cardiotoxins, myotoxins, hemostasis toxins, neurotoxins, and renal toxins, that can cause the following disorders containing rhabdomyolysis, acute kidney injury, paralysis of the extremities, compartment syndrome, wound necrosis, coagulation disorder, persistent mydriasis and respiratory distress, cardiac dysrhythmia [13].

Treatment by antivenom can reduces the envenomation effects on various systems (coagulation, central nervous system, the cardiovascular and gastrointestinal system; however, antivenom cannot reverse the effects of local tissue destruction or necrosis. ${ }^{13}$ Sever wound infections such as cellulitis and necroziting fasciitis following venomous snake bites, are not common; they have been reported in up to $30.8 \%$ of patients after a snake bite and require aggressive treatment $[3,4]$.

The common signs and symptoms of wound infections and cellulitis, such as erythema, inflammation, warmth and local pain may develop in the early hours to days of evolution. However, the risk factors developing from cellulitis to necrotizing fasciitis secondary to a snake bite provided have seldom been investigated [13].

One of the most important complications of snake bite with local envenoming are soft tissue infections. The snake venom with proteolytic properties causes extensive tissue destruction and devitalization, thus predisposing the wound to bacterial infection from the snake's indigenous oral flora. Although in patients with snake bite, bacteria are a major cause of wound infection, the role of prophylactic antibiotics to prevent their formation is discussible. However, the spectrum of bacteria from the venom and oral cavities of snakes vary with geographic area as well as with the species and the oral health of the snake, and these factors cannot easily be extrapolated to snakes in rest of the world [14]. 
Our reported patient was a 65 years old man who admitted with chest pain, tachycardia, fever, cold sweat, chills, frequent nausea, vomiting, left upper limb edema and decreased level of consciousness. After assessments and excluding the possibility of heart disease, infectious diseases and compartment syndrome, due to the burning sensation in the tip of the third finger of left upper limb after lifting a heavy object and the occurrence of hemorrhagic and non-hemorrhagic blisters in this limb with suspected snakebites, standard treatment with Anti-venom started for the patient and due to the rapid response with this treatment and the endemicity of the area in terms of snake bites, the diagnosis was definitive.

Hemorrhagic blisters often form at the site of the bite digits but may occur at any bite location or even in dependent areas distant from the bite. These blisters usually do not appear until hours after the bite. The tissue beneath these blisters is usually healthy, although large blisters can cause necrosis in their underlying tissue [2].

Coagulopathy, thrombocytopenia, or a combination of the two, may be present despite a paucity of other local or systemic effect. A decrease in platelet count, as well as decrease in fibrinogen with elevation of prothrombin time (PT), may be mild or moderate initially and may either remain so or continue to worsen for several days following the envenomation [2]. The standard Anti-venom treatment can reduce these toxic hemorrhagic and neurotoxic effects [6-8].

However, early usage of antivenom following snake bite, showed a trend toward a better local outcome within 12 hours. Prophylactic use of antibiotics was still controversial [7].

\section{Conclusion :}

It should be noted that wound infections caused by snake bites if not treated in a timely manner, can lead to complications such as necrotizing fasciitis, compartment syndrome, or superimposed bacterial infection, which require hospitalization, the use of broad-spectrum antibiotics, and emergency surgical procedures; and since blisters usually do not appear until hours after the bite, so in cases of local edema and swelling with hemorrhagic or non-hemorrhagic blisters of unknown cause, it is recommended to consider snake bite as a differential diagnosis and consult a clinical toxicology specialists .

\section{Acknowledgments}

Declared none.

\section{Authorships}

$\mathrm{ZZ}$ involved in interpretation and collecting of data, and editing the manuscript. LD, AZ involved in drafting first version of manuscript and editing. MB, MS involved in writing, editing and preparing the final version of manuscript. MF involved in critical revising. RT is responsible for collecting data and submitting the manuscript. All authors reviewed the paper and approved the final version of the manuscript.

\section{Conflict of Interest}

The authors confirm that this article content has no conflict of interest.

\section{References}

1. Dehghani, R., Fathi, B., Shahi, M.P. and Jazayeri, M. 2014. Ten years of snakebites in Iran. Toxicon. 90:291-298.

2. Rhua, A., A. Pizon, R. S. Hoffman, M. A. Howland, N. A. Lewin, L. S. Nelson, and L. R. Goldfrank. 2015. "Native (US) venomous snakes and lizards." Hoffman RS, Howland MA, Lewin NA, Nelson LS, Goldfrank LR. Goldfranks Toxicologic Emergencies. 10: 1537- 46.

3. Nadiyah, A., N. M. S. Azira, Z. Nazli, and M. Zeehaida. 2015. Post viper bite Pasteurella multocida necrotizing fasciitis complicates with septicaemia and renal failure. Tropical Biomedicine. 32 (4): 608-612. 
4. Liu, P.Y., Shi, Z.Y., Lin, C.F., Huang, J.A., Liu, J.W., Chan, K.W. and Tung, K.C. 2012. Shewanella infection of snake bites: a twelve-year retrospective study. Clinics. 67(5):431-435.

5. Otero, R., Gutierrez, J., Mesa, M.B., Duque, E., Rodriguez, O., Arango, J.L., Gómez, F., Toro, A., Cano, F., Rodriguez, L.M. and Caro, E. 2002. Complications of Bothrops, Porthidium, and Bothriechis snakebites in Colombia. A clinical and epidemiological study of 39 cases attended in a university hospital. Toxicon. 40(8): 1107-1114.

6. Chang, K.P., Lai, C.S. and Lin, S.D. 2007. Management of poisonous snake bites in southern Taiwan. The Kaohsiung journal of medical sciences. 23(10:511-518.

7. Chen, C.M., Wu, K.G., Chen, C.J. and Wang, C.M. 2011. Bacterial infection in association with snakebite: a 10-year experience in a northern Taiwan medical center. Journal of Microbiology, Immunology and Infection. 44(6):456-460.

8. Huang, L.W., Wang, J.D., Huang, J.A., Hu, S.Y., Wang, L.M. and Tsan, Y.T. 2012. Wound infections secondary to snakebite in central Taiwan. Journal of Venomous Animals and Toxins including Tropical Diseases. 18(3:272-276.

9. Shih YC, Ma H, Yeh FL, et al. 2006. Risk factors of surgical intervention in the management of venomous snakebite in northern Taiwan. J Plast Surg Assoc ROC. 15:367-376.

10. Hsu, C.P., Chuang, J.F., Hsu, Y.P., Wang, S.Y., Fu, C.Y., Yuan, K.C., Chen, C.H., Kang, S.C. and Liao, C.H. 2015. Predictors of the development of post-snakebite compartment syndrome. Scandinavian journal of trauma, resuscitation and emergency medicine. 23(1):97.

11. Wong, O.F., Lam, T.S., Fung, H.T. and Choy, C.H. 2010. Five-year experience with Chinese cobra (Naja atra)-related injuries in two acute hospitals in Hong Kong. Hong Kong Med J. 16(1): 36-43.

12. Cumpston, K.L. 2011. Is there a role for fasciotomy in Crotalinae envenomations in North America?. Clinical Toxicology. 49(5):351-365.

13. Tsai, Y.H., Hsu, W.H., Huang, K.C., Yu, P.A., Chen, C.L. and Kuo, L.T. 2017. Necrotizing fasciitis following venomous snakebites in a tertiary hospital of southwest Taiwan. International Journal of Infectious Diseases. 63:30-36.

14. Garg, A., Sujatha, S., Garg, J., Acharya, N.S. and Parija, S.C. 2009. Wound infections secondary to snakebite. The Journal of Infection in Developing Countries. 3(03):221-223. 